\title{
Amor, atitudes sexuais e índice de risco às DST em idosos
}

\section{Love, sexual attitudes and index risk to sexually transmitted diseases in the elderly}

\section{El amor, las actitudes sexuales y el índice de riesgo de ETS en personas mayores}

\author{
Luiz Antônio da Silva* \\ Universidade Salgado de Oliveira - UNIVERSO, Niterói, Rio de Janeiro, Brasil
}

\section{Lucia Helena de Freitas Pinho França**}

Universidade Salgado de Oliveira - UNIVERSO, Niterói, Rio de Janeiro, Brasil

\section{José Augusto Evangelho Hernandez***}

Universidade do Estado do Rio de Janeiro - UERJ, Rio De Janeiro, Rio de Janeiro, Brasil

\begin{abstract}
RESUMO
O crescente envelhecimento populacional e os temas relativos à velhice têm recebido destaque nas ciências sociais e da saúde. Este estudo investigou as possíveis relações entre as percepções do amor e das atitudes sexuais e o risco de contágio das Doenças Sexualmente Transmissíveis (DST) na velhice. Foram avaliados 352 idosos cognitivamente saudáveis do Estado do Rio de Janeiro, que responderam a Escala Triangular do Amor de Sternberg (ETAS), a Brief Sexual Attitudes Scale (BSAS) e um questionário sociodemográfico que também avaliou o grau de risco às DST nas relações sexuais dos idosos. A Análise Fatorial Exploratória revelou fraquezas psicométricas na versão adaptada da BSAS que invalidaram a maior parte dos itens e fatores, excetuando-se o fator Permissividade. Foram apurados níveis significamente maiores de Permissividade nos homens, em comparação com as mulheres. Os resultados revelaram que, quanto maior o nível global de Amor, maior o risco às DST para as mulheres, que adotam uma orientação do sexo mais relacional e centrada na pessoa; os homens adotaram orientação mais recreacional e centrada no corpo; quanto maior a Idade, menor o nível de Permissividade e menor será o risco às DST. Recomenda-se a replicação desta pesquisa em outras amostras de idosos.
\end{abstract}

Palavras-chave: amor, atitudes sexuais, doenças sexualmente transmissíveis, idosos.

\section{ABSTRACT}

The growing aging population and the issues related to elderly have received prominence in the social sciences and health. This study investigated the possible relationship between perceptions of love and sexual attitudes and 
the contagion risk of Sexually Transmitted Diseases (STD) in old age. They evaluated 352 healthy older participantes of the State of Rio de Janeiro, who answered Triangular Scale Sternberg Love (ETAS), the Brief Sexual Attitudes Scale (BSAS) and a sociodemographic questionnaire also assessed the degree of risk to STDs during sexual intercourse of the elderly. The Exploratory Factor Analysis revealed psychometric weaknesses with the adapted version of BSAS that invalidated most of the items and factors, except for the permittivity factor. Significantly higher levels were determined in permissiveness for men compared to women. The results revealed that the higher the overall level in ETAS the higher is the risk of STDs for women; women adopt the guidance guidance in the relational-centered sex in person while men, more recreational and body-centered; the higher the age and lower level of permissiveness the lower is the risk of STDs, for men. It is suggested that future studies can replicate this research with other samples of elderly.

Keywords: love, sexual attitudes, sexually transmitted diseases, elderly.

\section{RESUMEN}

El creciente envejecimiento de la población y las cuestiones relacionadas con la vejez han recibido prominencia en las ciencias sociales y de la salud.Este estudio investigó la posible relación entre las percepciones de amor y actitudes sexuales y el riesgo de contagio de enfermedades de transmisión sexual (ETS) en la vejez. Ellos evaluaron 352 sujetos sanos de edad avanzada del estado de Río de Janeiro que respondieron laTriangular Escala Sternberg Love (ETAS), el Brief Sexual Attitudes Scale (BSAS) y un cuestionario sociodemográfico que también evaluaron el grado de riesgo de ETS durante las relaciones sexuales de las personas mayores. El análisis factorial exploratorio reveló debilidades psicométricas con la versión adaptada del BSAS que invalidó la mayor parte de los elementos y factores, excepto por el factor de permisividad. Significativamente mayores niveles se determinaron en la permisividad para los hombres en comparación con las mujeres. Los resultados revelaron que: cuanto mayor es el nivel general de ETAS mayor es el riesgo de ETS para la mujer; que adoptan la orientación en el sexo-relacional centrado en la persona, mientras que los hombres, más lúdico y centrada en el cuerpo; cuanto mayor sea la edad, menor el nivel de la permisividad e menor el riesgo de ETS, para los hombres. Se sugiere la replicación desta investigación con otras muestras de ancianos.

Palabras clave: amor, actitudes sexuales, enfermedades de transmisión sexual, adultos mayores.

\section{Introdução}

O objetivo deste estudo foi verificar a relação de variáveis interpessoais como o amor e atitudes sexuais com o comportamento de prevenção às Doenças Sexualmente Transmitidas de pessoas idosas no contexto dos relacionamentos íntimos. Pesquisas anteriores indicam que questões interpessoais podem estar associadas com comportamento de prevenção (Adler \& Hendrick, 1991).

De acordo com a Pesquisa Nacional por Amostra de Domicílios (PNAD) do Instituto Brasileiro de Geografia e Estatística (2014), pessoas com idade igual ou superior a 60 anos representavam $13 \%$ 
da população brasileira em 2013. A estimativa é que este número atinja os $29 \%$ em 2050, porém, mesmo com esses dados expressivos, pouco se conhece sobre o amor e o sexo no processo do envelhecimento no Brasil. Além disso, Dorneles Neto, Nakamura, Cortez e Yamaguchi (2015) realizaram uma revisão sistemática nas bases de dados acerca de publicações que abordaram a evolução das DSTs em idosos no Brasil no período de 1980 a 2013. Os pesquisadores concluíram que essa faixa etária estava completamente fora das políticas públicas de promoção de saúde no contexto das DSTs.

Garret (2014) realizou uma revisão da literatura de 1995-2013 relativa às barreiras psicossociais que exercem fortes influências sobre a intimidade sexual de pessoas com idades entre 75-85 anos. Essas influências incluíram: a falta de política social positiva, a falta de pesquisa, a disponibilidade do parceiro, as imagens negativas da mídia, os fatores psicológicos e de relacionamentos e as dificuldades na interação com profissionais de saúde. As atitudes estereotipadas sobre intimidade sexual de pessoas idosas continuam a ser uma norma cultural. Um valor elevado de desempenho sexual é colocado como padrão de ouro, o que pode definir expectativas e medidas irrealistas para os idosos.

Neste estudo, o amor foi abordado por meio da Teoria Triangular do Amor desenvolvida por Sternberg (1986), que foi construída mediante a análise crítica dos achados anteriores da psicologia clínica, social e da personalidade, até chegar a uma estrutura suficientemente completa para cobrir aspectos essenciais da complexidade do amor. Considerada uma das teorias mais abrangentes acerca das relações amorosas, tornou-se muito difundida na pesquisa psicológica dos relacionamentos íntimos nas últimas décadas. Esta teoria prevê alguns componentes importantes para o amor: Paixão, Intimidade e Decisão/Compromisso.

O componente Intimidade ocupa o topo do vértice do triângulo, metáfora geométrica adotada por Sternberg (1986). Embora todos os componentes tenham seu valor e possam se apresentar de modo único ou em conjunto, a Intimidade representa o desejo de promover o bem estar da pessoa amada, a felicidade por compartilhar experiências de vida, o respeito mútuo, a compreensão mútua em momentos adversos, a compreensão e sintonia íntimas, o apoio emocional mútuo, a comunicação profunda e o reconhecimento do valor da pessoa amada na sua vida.

A Paixão envolve a excitação física e emocional, as sensações românticas, a atração física, o desejo de estar junto e a satisfação sexual mútua. Em alguns relacionamentos amorosos, ela pode ser fundamental, em outros, secundária. É a consumação do sentimento em ações que se manifestam pelo desejo constante e intenso de ter e estar unido com o outro (Sternberg, 1988). 
O componente Decisão/Compromisso é o vértice direito do triângulo e compreende duas fases, a Decisão de que se ama alguém e o Compromisso, interesse em um relacionamento continuado, em manter a união e a amar o outro. A Decisão/Compromisso representa o reconhecimento e a manutenção do amor (Sternberg, 1988).

Nos relacionamentos, os três componentes do amor não têm sempre - mesmo peso e variações combinadas dos mesmos podem configurar tipos diferentes de amor. Os níveis de Intimidade, Paixão e Decisão/Compromisso podem mudar com o tempo e alterar 0 relacionamento. Normalmente, a Paixão é mais forte no começo da relação. Com o sexo regular, ela se estabiliza numa forma menos urgente. A Intimidade aumenta continuamente, seu crescimento torna-se mais lento e, por fim, estável. Na continuidade da relação, os amantes tornam-se mais previsíveis um para o outro. Geralmente a Decisão/Compromisso começa gradualmente, se acelera, e se o relacionamento perdura por um longo período, a sensação de compromisso se estabiliza (Sternberg, 1988).

$\mathrm{Na}$ literatura são raros os estudos que abordam o amor entre pessoas idosas. Rocha e Hernandez (2002) compararam grupos de adultos jovens (de 18 a 25 anos de idade) e idosos (maiores de 60 anos), que estavam em relações amorosas estáveis. Nos resultados, os níveis do componente Decisão/Compromisso relatados pelos idosos foram significativamente mais elevados do que os dos adultos jovens. Entre os idosos, os níveis de Paixão foram maiores para homens do que para mulheres. Em Villar, Villamizar e López-Chivrall (2005), a experiência amorosa relatada pelos idosos revelou níveis de Intimidade e Paixão mais elevados para os homens do que para as mulheres. Em Decisão/Compromisso não houve diferença significativa entre os sexos e os níveis foram maiores do que para os outros dois componentes do amor.

As transformações biológicas que ocorrem durante a vida adulta sugerem que as pessoas idosas, em geral, tendem a apresentar níveis mais baixos de Paixão na comparação com as mais jovens. Contudo, alguns estudos mostraram que a diminuição da Paixão, com o aumento da idade, pode ser pequena (Ahmetoglu, Swami, \& Chamorro-Premuzic, 2010). Os idosos podem continuar a ter relações amorosas satisfatórias (Rokach \& Neto, 2005) e até mesmo os aspectos eróticos da Paixão podem ser experimentados com intensidade (Neto, 2012).

De acordo com Adler e Hendrick (1991), é esperado que um tipo de amor mais carregado emocionalmente como a Paixão, seja inversamente relacionado com comportamento de prevenção. Práticas sexuais, por sua própria natureza, implicariam responsabilidade e prevenção. $O$ investimento emocional contido na Comunhão também estaria relacionado com maior responsabilidade com o parceiro sexual e, consequentemente, com a prevenção. 
As atitudes sexuais abordadas neste estudo foram definidas por Hendrick e Hendrick (1995) como cognições e comportamentos que são influenciados por fatores variados, que vão da prática sexual e da interpretação do sujeito sobre o que esta representa, passando por questões de gênero e de cultura. Hendrick, Hendrick, Slapion-Foote e Foote (1985), investigando as atitudes de universitários com relação a atividade sexual, mostraram que, em geral, os homens apresentam atitudes sexuais mais permissivas se comparados às mulheres, interpretando o sexo como algo físico. As mulheres, por sua vez, tendem a experimentar o sexo como uma experiência emocional, preferem a estabilidade do amor-amizade, especialmente pela presença de parceria e intimidade. Por outro lado, os homens preferem o amor no estilo do jogo de conquista. Oliver e Hyde (1993) revisaram a literatura das diferenças sexuais na permissividade sexual geral e encontraram um efeito de tamanho médio $(d=0,57)$ no qual homens foram mais permissivos do que as mulheres. Petersen e Hyde (2010) encontraram o mesmo resultado, mas a diferença foi menor $(d=0.21)$, e concluíram que a mesma tem diminuído ao longo das últimas décadas.

O uso do preservativo é uma das medidas consagradas para a prevenção de DSTs, mas poucas pesquisas abordam o seu uso entre idosos e os fatores que levam à sua adoção ou dispensa. No estudo de Abdo, Oliveira, Scanavino e Martins (2006) com pessoas com mais de 60 anos de idade, cerca de $18 \%$ dos homens e $13 \%$ das mulheres dispensaram o uso do preservativo em seus relacionamentos sexuais. Laurentino et al., (2006) comentaram que, entre idosos, há uma atitude sexual de maior susceptibilidade ao contágio por DSTs em razão do comportamento sexual comum de pouca orientação e prevenção.

Laroque et al. (2011) mostraram que os idosos tinham informações sobre as possibilidades de contágio, mas, mesmo assim, poucos aderiam ao uso de preservativos. Entre os vários motivos para esse comportamento, está o fato de que as experiências sexuais na juventude e na vida adulta não tenham ocorrido na perspectiva da saúde. Entre os fatores que protagonizavam as práticas sexuais estavam o exercício sexual associado à culpa, a pressão social e moral e, especialmente entre as mulheres, a condenação do prazer sexual. Em razão disso, os comportamentos ligados à sexualidade foram incorporados de modo mais lento, ou não foram, pela população idosa (Bodley-Tickell et al., 2008; Pascual, 2002).

Neste estudo, foram verificadas as propriedades psicométricas da adaptação da Brief Sexual Attitudes Scale (Hendrick, Hendrick, \& Reich, 2006) com os escores de idosos brasileiros. Além disso, foram testadas as relações do amor, das atitudes sexuais e do 
comportamento de risco às DSTs, representado pelo uso ou não do preservativo.

\section{Método}

\section{Participantes}

A amostra foi composta por 352 pessoas com idade entre 60 e 90 anos $(M=67,4 ; D P=6,18)$, cognitivamente saudáveis. Do total dos respondentes, $182(51,7 \%)$ eram do sexo feminino e $170(48,3 \%)$, do masculino. Quanto ao estado civil: $181(51,4 \%)$ casados, $28(8 \%)$ união estável, $56(15,9 \%)$ viúvos, $38(10,8 \%)$ solteiros, $28(8 \%)$ separados, $17(4,8 \%)$ divorciados, $2(0,6 \%)$ desquitados e $2(0,6 \%)$ namorando. No que tange à escolaridade: $63(17,9 \%)$ curso primário, $58(16,5 \%)$ ginásio, $121(34,4 \%)$ segundo grau e $110(31,3 \%)$ superior completo. No que diz respeito à frequência sexual: 100 $(28,4 \%)$ não tiveram relação sexual no último ano, $18(5,1 \%)$ tiveram menos de seis por ano, $4(1,1 \%)$, de seis a 10 por ano, 22 $(6,3 \%)$, uma vez por mês, $47(13,4 \%)$, a cada 15 dias, $110(31,3 \%)$, uma ou duas vezes por semana, $49(13,9 \%)$, três a seis vezes por semana e $2(0,6 \%)$, todos os dias.

\section{Instrumentos}

Foi utilizada a ETAS (Sternberg, 1997), que é composta pelos fatores Paixão, Intimidade e Decisão/Compromisso, na versão brasileira adaptada por Hernandez (1999). O instrumento possui 45 itens igualmente distribuídos entre as três subescalas. Para responder à medida, os participantes usaram uma escala tipo Likert de nove pontos, onde 1 é "nada verdadeiro", 5 é "moderadamente verdadeiro" e 9 é "totalmente verdadeiro", os pontos intermediários indicaram níveis intermediários de sentimentos.

A Escala Triangular do Amor de Sternberg (ETAS) (Sternberg, 1997) foi criada para representar, no plano empírico, o construto do amor por meio de três fatores distintos. Vários estudos têm encontrado esta estrutura hierárquica multifatorial para a ETAS (Cassepp-Borges \& Pasquali, 2012; Cassepp-Borges, \& Teodoro, 2007; Hernandez, 1999, 2015). Entretanto, Graham (2011) realizou uma meta-análise envolvendo 19.387 indivíduos e abrangendo 40 anos de investigações sobre o amor, e os itens representativos dos três componentes da ETAS saturaram suas cargas fatoriais em um único fator. Segundo o pesquisador, a Teoria Triangular do Amor forneceria um modelo elegante combinando Paixão, Intimidade e Decisão/Compromisso, mas o teste sugeriu que essas dimensões não seriam distintas. 
Hernandez (2016) também encontrou evidências de uma estrutura unifatorial para a escala.

Hendrick e Hendrick (1987) concluíram a construção da Sexual Attitudes Scale com quatro subescalas, Permissividade (sexo casual e sem culpa), Práticas Sexuais (responsabilidade sexual), a Comunhão (sexualidade idealística) e a Instrumentalidade (sexualidade utilitária e egoísta). Porém Hendrick, Hendrick e Reich (2006) definiram uma versão do instrumento denominada Brief Sexual Attitudes Scale (BSAS), na qual a subescala Práticas Sexuais foi reformulada para Controle de Natalidade.

Neste estudo foi usada a adaptação brasileira da BSAS, que foi traduzida do inglês por dois psicólogos bilíngues e comparada com as versões portuguesas de Alferes (1999) e Felipe (2012). A BSAS possui 23 itens distribuídos em quatro subescalas, Permissividade, Controle de Natalidade, Comunhão e Instrumentalidade. $\mathrm{Na}$ atual adaptação, os três itens da subescala original Controle de Natalidade foram completamente reformulados para representar o conteúdo prevenção, uma vez que o uso do preservativo na velhice é objeto desta pesquisa. O nome da subescala foi mudado para Prevenção. Para responder a BSAS, os participantes usaram uma escala tipo Likert de cinco pontos, onde 1 é "concordo fortemente", 3 é "não concordo, nem discordo" e 5 é "discordo fortemente".

\section{Coleta de dados}

Este projeto foi submetido e aprovado pelo comitê da universidade a qual está vinculado no dia 29/11/2014, sob o número CAAE 39750714.1.00005289. Todos os participantes foram informados sobre os objetivos da pesquisa, leram e assinaram o Termo de Consentimento Livre e Esclarecido. Os dados foram coletados de forma coletiva em instituições, organizações e programas de terceira idade. Em geral, os idosos tiveram condições de responder aos questionários, porém alguns apresentaram dificuldades e necessitaram da ajuda do pesquisador. Em decorrência disso, o tempo de preenchimento dos questionários variou de 40 a 100 minutos.

\section{Análise de dados}

Os dados foram analisados no programa Factor 9.3.1 (Lorenzo-Seva \& Ferrando, 2013) por meio de Análise Fatorial Exploratória (AFE) para verificação da estrutura fatorial da BSAS adaptada. No SPSS, versão 20, foram utilizadas técnicas estatísticas descritivas, Teste $t$ de Student, Coeficiente de Correlação de Pearson e Análise de Regressão Múltipla para analisar as relações entre as variáveis investigadas: amor, atitudes sexuais e risco às DSTs. 


\section{Resultados}

\section{Análise Fatorial da BSAS}

Do total de escores coletados, apenas 0,4\% estavam ausentes e foram repostos pela média. Ficou evidente a anormalidade multivariada da distribuição dos dados, porém, na estatística univariada, a assimetria foi $< \pm 2$ e a curtose $< \pm 7$, o que não é numa violação extrema da normalidade (Finney \& DiStefano, 2006). O item 11 ("Uma relação sexual é aceitável") da subescala Permissividade foi previamente excluído da análise fatorial, pois foi apresentado para os respondentes com erro de digitação, o que alterou o conteúdo do mesmo. A formulação correta seria "Uma relação sexual casual é aceitável".

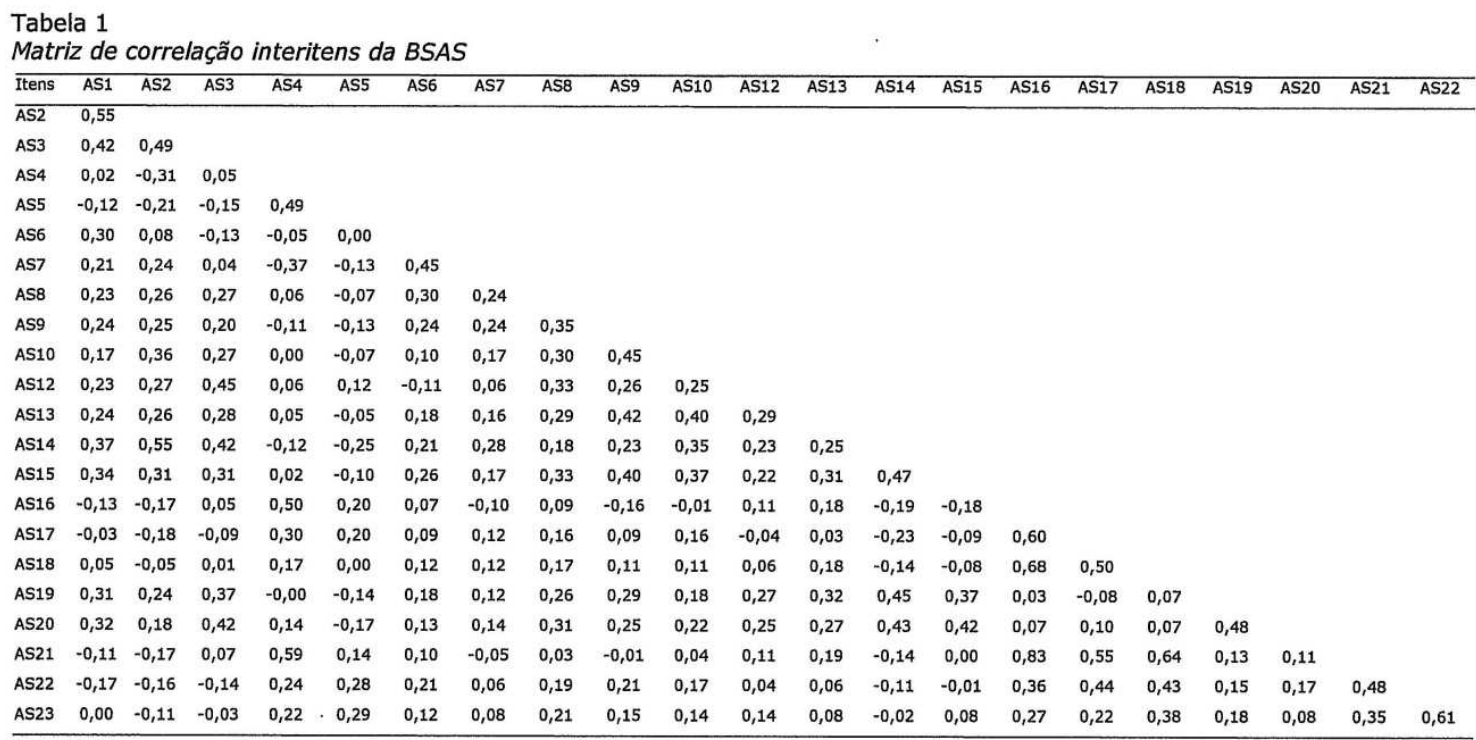

A adequação dos dados da análise fatorial da BSAS com 22 itens foi verificada mediante o teste $\mathrm{KMO}$, que forneceu um valor de 0,76, considerado aceitável. $\mathrm{O}$ teste de esfericidade de Bartlett apresentou os seguintes valores: $X^{2}{ }_{(231)}=1.880,89 ; p<0,001$, que permitiram rejeitar a hipótese nula e confirmaram a utilização do método de análise para os dados coletados. A matriz de correlação de dados analisada pode ser observada na Tabela 1.

A Análise Fatorial Exploratória (AFE) com método de extração Unweighted Least Squares (ULS) e rotação promin (Lorenzo-Seva, 2013) foi executada e os resultados revelaram sete fatores com autovalores > 1,0. Mas, a Análise Paralela de Horn (1965) recomendou a retenção de quatro fatores (Tabela 2 ) coerentes com o modelo teórico de Hendrick, Hendrick e Reich (2006).

Uma nova AFE para quatro fatores foi executada. Na análise da matriz de fator rotada, considerando cargas fatoriais $\geq 0,32$ (Laros, 
2005), observou-se que, no Fator 1 , carregaram todos os itens teoricamente designados para a dimensão Permissividade e mais três itens da dimensão Instrumentalidade (que também carregaram mais fracos no Fator 4). No fator 2, carregaram os itens 16 e 21 da Prevenção e também dois itens da Comunhão. No fator 3 , carregaram os itens 22 e 23 da Comunhão e um item da Prevenção (que também carregou fraco no Fator 4). No fator 4, carregaram os itens 6 e 7 da Instrumentalidade e mais um item da Prevenção. Foi tomada a decisão de excluir esses sete itens que carregaram em fatores não determinados no modelo teórico ou apresentaram cargas fatoriais fracas $(<0,32)$ divididas entre os fatores (Laros, 2005; Lloret-Segura, Ferreres-Traver, Hernández-Baeza, \& Tomás-Marco, 2014).

Tabela 2

Autovalores Empíricos e Aleatórios da Análise Paralela

\begin{tabular}{|c|c|c|c|}
\hline Fatores & Autovalor Empírico & Autovalor Aleatório & $95^{\circ}$ Autovalor Aleatório \\
\hline 1 & 3,99479 & 1,47793 & 1,56023 \\
\hline 2 & 3,04027 & 1,39306 & 1,44761 \\
\hline 3 & 1,63883 & 1,33398 & 1,37966 \\
\hline 4 & 1,43307 & 1,28130 & 1,32428 \\
\hline 5 & 1,14971 & 1,23345 & 1,27033 \\
\hline
\end{tabular}

Nota. 500 matrizes aleatórias de Pearson.

Novamente foi realizada uma AFE com mesmo método e rotação para os 15 itens restantes da BSAS (Tabela 3). A variância comum explicada pelos quatro fatores extraídos foi de $74 \%$ da variância comum total. Como pode ser observado na Tabela 3, as dimensões Comunhão, Instrumentalidade e Prevenção ficaram fragilizadas com a perda de itens, restando apenas dois em cada uma delas. Autores como Lloret-Segura et al., (2014) recomendaram que o número mínimo de itens por fator seja de três ou quatro, especialmente quando o tamanho da amostra for grande. Embora a dimensão Prevenção tenha apresentado um alfa ótimo, teve uma perda importante de conteúdo referente à responsabilidade masculina ("O homem deve partilhar a responsabilidade pela prevenção"). 
Tabela 3

Matriz Rotada de Cargas Fatoriais, Comunalidades e Alfas de Cronbach da BSAS

\begin{tabular}{|c|c|c|c|c|c|c|}
\hline & & & Fat & & & $h^{2}$ \\
\hline & - & 1 & 2 & 3 & 4 & \\
\hline$\overline{6}$ & A relação sexual é melhor quando nos focamos no nosso próprio & & & . & & \\
\hline & prazer. & 0,89 & 0,05 & $-0,29$ & 0,01 & 0,69 \\
\hline 7 & A relação sexual serve, em primeiro lugar, para retirar prazer & & & & & \\
\hline & da outra pessoa. & 0,43 & 0,02 & 0,01 & 0,01 & 0,20 \\
\hline 16 & A prevenção faz parte de uma sexualidade responsável. & $-0,01$ & 0,93 & $-0,01$ & $-0,10$ & 0,96 \\
\hline 21 & A mulher deve partilhar a responsabilidade pela prevenção. & 0,02 & 0,44 & 0,01 & 0,17 & 0,50 \\
\hline 3 & É possível apreciar uma relação sexual com uma pessoa sem & & & & & \\
\hline & gostar muito dela. & $-0,17$ & 0,05 & 0,75 & $-0,14$ & 0,49 \\
\hline 2 & É aceitável manter relacionamentos sexuais com mais de uma & & & & & \\
\hline & pessoa ao mesmo tempo. & 0,15 & 0,02 & 0,48 & $-0,22$ & 0,33 \\
\hline 19 & $\begin{array}{l}\text { Relações sexuais casuais, de uma noite apenas, conseguem ser } \\
\text { muito satisfatórias. }\end{array}$ & 0,11 & $-0,05$ & 0,49 & 0,18 & 0,33 \\
\hline $\begin{array}{l}14 \\
15\end{array}$ & $\begin{array}{l}\text { Gostaria de ter relações sexuais com muitos (as) parceiros (as). } \\
\text { As melhores relações sexuais acontecem quando não existe }\end{array}$ & 0,26 & $-0,08$ & 0,47 & $-0,06$ & 0,33 \\
\hline & compromisso. & 0,21 & $-0,12$ & 0,47 & 0,06 & 0,28 \\
\hline 1 & $\begin{array}{l}\text { Não preciso estar comprometido (a) com uma pessoa para ter } \\
\text { relações sexuais com ela. }\end{array}$ & 0,26 & $-0,01$ & 0,43 & $-0,20$ & 0,34 \\
\hline 20 & $\begin{array}{l}\text { A vida teria menos problemas se as pessoas pudessem ter } \\
\text { relações sexuais de forma mais livre. }\end{array}$ & 0,07 & $-0,01$ & 0,50 & 0,13 & 0,29 \\
\hline 12 & A relação sexual como uma simples troca de favores é aceitável & & & & & \\
\hline & se ambas as pessoas aceitarem. & $-0,17$ & 0,07 & 0,49 & 0,09 & 0,23 \\
\hline 13 & $\begin{array}{l}\text { É aceitável que a relação sexual seja apenas uma agradável } \\
\text { sensação física. }\end{array}$ & 0,13 & 0,12 & 0,32 & 0,04 & 0,18 \\
\hline 22 & A relação sexual entre pessoas muito apaixonadas é o expoente & & & & & \\
\hline & máximo das relações humanas. & $-0,01$ & $-0,01$ & $-0,04$ & 0,80 & 0,63 \\
\hline 23 & $\begin{array}{l}\text { A relação sexual é uma experiência intensa, quase } \\
\text { arrebatadora. }\end{array}$ & $-0,01$ & 0,03 & 0,05 & 0,57 & 0,35 \\
\hline & Alfa de Cronbach & 0,76 & 0,96 & 0,78 & 0,72 & \\
\hline & Variância comum explicada & 1,3 & 1,5 & 2,2 & 1,2 & \\
\hline & Variância comum explicada (\%) & 21,2 & 24,4 & 35,8 & 19,5 & \\
\hline & Correlação & & & & & \\
\hline & F2 & 0,02 & & & & \\
\hline & F3 & 0,37 & 0,07 & & & \\
\hline & F4 & 0,21 & 0,37 & 0,06 & & \\
\hline
\end{tabular}

Notas. Método de extração: Unweighted Least Squares. Método de rotação: Promin.

F1 = Instrumentalidade; F2 = Prevenção; F3 = Permissividade; F4 = Comunhão

Apenas a dimensão Permissividade se manteve mais intacta (nove itens), perdendo apenas um item e apresentando aceitável consistência interna. Diante deste quadro, decidiu-se utilizar apenas a 
subescala Permissividade da BSAS nas análises estatísticas subsequentes.

Neste estudo, os alfas de Cronbach foram de 0,98 para a ETAS (medida global, 45 itens), 0,94 para o fator Paixão (15 itens), 0,95 para a Intimidade (15 itens) e 0,96 para a Decisão/Compromisso (15 itens). Estes valores foram semelhantes aos encontrados em pesquisas nacionais anteriores (Cassepp-Borges \& Pasquali, 2012; Cassepp-Borges \& Teodoro, 2007, Hernandez, 1999).

\section{Amor, Atitudes Sexuais e Risco de DST}

A maioria dos participantes $(96,6 \%)$ afirmou ser favorável ao uso do preservativo. No entanto, as medidas referentes ao uso efetivo de preservativo nas relações sexuais com a (0) esposa (0) $(M=1,22$; $D P=1,62)$, com a parceira (o) sem compromisso $(M=1,51 ; D P=$ $2,13)$ ou com a (o) namorada (o) $(M=1,41 ; D P=2,04)$ apresentaram valores médios baixos, indicativos de não utilização dessa prevenção. Ou seja, apesar da declaração de atitude favorável ao uso de preservativos, não costumavam usá-los.

A distribuição geral dos escores da variável Índice de Risco às DSTs apresentou pequena assimetria, mas não foi identificada a presença de valores extremos. Para as mulheres $(n=182)$, foram identificadas correlações lineares significativas $(p<0,05)$ entre as variáveis Paixão, Intimidade, Compromisso e a ETAS (medida global) e a variável Índice de Risco às DSTs, mas as correlações com as variáveis Idade e Permissividade não foram significativas. Como esperado, as variáveis Paixão, Intimidade e Decisão/Compromisso da ETAS apresentaram multicolinearidade, portanto, na Análise de Regressão foi utilizada a medida global do amor (ETAS). Diversos pesquisadores têm considerado a ETAS como unidimensional (Acker \& Davis, 1992; Carreño \& Serrano, 1995; Gouveia, Fonseca, Cavalcanti, Diniz, \& Dória, 2009; Graham, 2011; Hendrick \& Hendrick, 1989; Hernandez, 2016).

Para mulheres ( $n=182$ ), o Coeficiente de Correlação de Pearson revelou uma relação inversa entre a ETAS e o Risco às DSTs, $r=$ $0,24, p<0,001$, indicando um pequeno efeito sobre a variável critério (explicando $5 \%$ da variância). Neste caso, quanto maior o escore na medida global do amor, maior o risco (valores menores do Índice de Risco representaram graus maiores de riscos de DSTs).

Para os homens ( $n=170)$, as correlações significativas foram entre Idade, Permissividade e Índice de Risco às DSTs. Não houve correlações significativas da ETAS e suas subescalas com o Índice de Risco às DSTs. Uma Análise de Regressão Múltipla, método Stepwise, foi executada para as variáveis explicativas Idade e Permissividade e variável critério Índice de Risco às DSTs. A principal preditora do risco para os homens foi a Idade, quanto maior a Idade menor o risco, 
sendo responsável por $5 \%$ da variação dos dados. Também foi encontrada uma correlação inversa entre a Idade e a frequência de relações sexuais, $r=-0,28, p<0,01$. Um segundo modelo da regressão incluiu a Permissividade junto com a Idade e ampliou a explicação da variância para 8\% (Tabela 4).

Tabela 4

Regressão Múltipla Stepwise para o Sexo Masculino

\begin{tabular}{llccccc}
\hline Modelos & Preditores & $R$ & $R^{2}$ & Beta padronizado & $F$ & $p$ \\
\hline 1 & Idade & 0,21 & 0,05 & 0,21 & 8.020 & 0,005 \\
2 & Idade & 0,28 & 0,08 & 0,21 & 7.352 & 0,001 \\
& Permissividade & & & $-0,19$ & & \\
\hline
\end{tabular}

Nota. Variável critério Índice de Risco às DSTs.

O Teste $t$ de Student para amostras independentes, $t_{(350)}=6.198, p$ $<0,001$, revelou diferença estatística significativa entre as médias de homens $(3,1)$ e de mulheres $(3,7)$ em Permissividade. Neste caso, valores menores indicaram mais Permissividade, portanto os homens mostraram-se mais permissivos do que as mulheres. O tamanho do efeito da variável sexo sobre a Permissividade foi $(d=0,68)$ considerado médio (Cohen, 1988).

O Teste $t$ de Student para amostras independentes apurou uma diferença estatística significativa entre as médias de homens e mulheres no Índice de Risco às DSTs, $t_{(350)}=3.860, p<0,001, d=$ 0,41 . Os homens apresentaram um Índice de Risco às DSTs maior do que as mulheres com tamanho do efeito médio (Cohen, 1988).

\section{Discussão}

Nesta pesquisa a AFE dos escores da BSAS não obteve o resultado esperado para a validade fatorial. Vários itens da escala não apresentaram a saturação de suas cargas fatoriais nos fatores da forma prevista no modelo teórico. Assim, as propriedades psicométricas do instrumento ficaram abaixo do condição adequada para uma medida válida de atitudes sexuais com idosos.

Hendrick, Hendrick e Reich (2006) encontraram evidências de fortes propriedades psicométricas para o instrumento. Porque os escores da BSAS não geraram evidências de validade suficientes para a medida nesta pesquisa atual? A explicação para isso pode residir nas características da presente amostra (idosos), diferente das anteriores (Hendrick \& Hendrick, 1987; Hendrick et al., 1985; Hendricket al., 2006), em que participaram estudantes universitários com idades médias em torno dos 20 anos. A média de idade dos participantes 
atuais ficou em torno dos 67 anos e, além disso, quase $70 \%$ dos mesmos possuía escolaridade em nível de ensino médio ou inferior. É possível que a idade e escolaridade dos respondentes tenham influenciado suas respostas na BSAS.

Os hormonios sexuais declinam para homens e mulheres com 0 avanço da idade. A redução da elasticidade e lubrificação vaginal, para as mulheres, poderá prejudicar a atividade sexual. No entanto, há pouca evidência de que as mulheres percam o desejo sexual. Por outro lado, há alguma especulação de que o homem possa ter um fenomeno similar, a andropausa, mas também há poucas evidências disso. Assim, o desejo sexual poderá continuar existindo para ambos (Petersen \& Hyde, 2011).

$\mathrm{Na}$ análise dos dados, os homens apresentaram níveis mais elevados em permissividade do que as mulheres, resultado também encontrado nas pesquisas anteriores (Hall, Hendrick, \& Hendrick, 1991; Hendrick \& Hendrick, 1986, 1987, 1995; Hendrick et al., 1985; Oliver \& Hyde, 1993; Petersen \& Hyde, 2010). A Permissividade também apareceu como fator preditor de risco às DSTs para os homens, mas não para mulheres. Em geral, os homens apresentaram maior risco às DSTs do que as mulheres.

As diferenças em atitudes e comportamentos sexuais entre homens e mulheres têm sido explicadas por importantes abordagens teóricas. A Psicologia Evolucionista postula que as mesmas são decorrentes de diferentes estratégias evolucionárias adotadas por cada um dos sexos para garantir a preservação dos genes (Buss, 1998). Os homens teriam muitas parceiras e relacionamentos sexuais casuais na tentativa de aumentar as chances de sobrevivência de sua herança genética. Por outro lado, as mulheres seriam mais seletivas e prefeririam relacionamentos sexuais de longa duração nos quais os parceiros protegeriam e ajudariam a cuidar das crianças (Petersen \& Hyde, 2011).

A Teoria Socio-Cognitiva (Bussey \& Bandura, 1999) sustenta a existência de um duplo padrão internalizado na determinação de atitudes e comportamentos sexuais que serão imitados por homens e mulheres. O duplo padrão sexual supõe que sexo casual e múltiplos parceiros sexuais são aceitáveis para homens, mas não para mulheres (Milhausen \& Herold, 2001). A regulação de comportamentos sexuais permissivos em mulheres se daria pela punição das mesmas (Crawford \& Popp, 2003; Kreager \& Staff, 2009), tais como uma simples desaprovação pelos pares. Isso explicaria a tendência dos homens a serem mais permissivos sexualmente do que as mulheres.

Como na maioria das sociedades no mundo, os homens têm mais poder do que as mulheres, e a eles é permitido que sejam mais permissivos sexualmente. A Teoria Estrutural Social (Eagly \& Wood, 1999) sustenta que diferenças nas atitudes e comportamentos 
sexuais humanos diferem conforme o poder e a diferenciação de tarefas para cada um dos sexos.

Pesquisadores, por meio de meta-análises, têm investigado diferenças de gênero especificamente em atitude sexuais. Oliver e Hyde (1993) encontraram um efeito médio para as diferenças sexuais na escalas de permissividade sexual, no qual os homens foram mais permissivos do que as mulheres, resultado semelhante ao do estudo atual. Petersen e Hyde (2010) encontraram um efeito menor e sugeriram que essa diferença tem diminuído com o tempo.

A idade também se revelou um fator preditivo da diminuição do risco às DSTs para os homens desta pesquisa. Isso se explicaria pela diminuição da atividade sexual, que se apresentou associada ao aumento da idade neste grupo, conforme a correlação encontrada.

Para as mulheres, o escore global na ETAS foi revelado como preditor do risco às DSTs, quanto mais elevado esse escore maior o risco. Conforme Hendrick e Hendrick (2002), as mulheres entenderiam o desejo sexual como amor, enquanto para os homens seria atividade sexual. As mulheres adotam uma orientação mais relacional e centrada na pessoa, enquanto os homens, mais recreacional e centrada no corpo. Possivelmente as mulheres envolvidas pelos sentimentos do amor se tornariam menos precavidas e, portanto, mais expostas aos riscos de DSTs em suas relações sexuais.

Em parte, o pouco uso do preservativo relatado neste estudo também pode ter associação com a despreocupação com a natalidade nos relacionamentos sexuais entre os idosos (Bodley-Tickell et al., 2008; Gradim, Sousa, \& Lobo, 2007; Trudel et al., 2008). Ainda que exista farta disseminação de informações sobre o risco de contágio por doenças, a negligência ocorre em grande parte dos casos (Abdo et al., 2006). Isso pode estar baseado no aprendizado formado na vida jovem e adulta, quando as experiências sexuais se iniciaram, e não houve a demanda de autocuidado para incorporação do hábito da prevenção (Pascual, 2002). Há uma variada disponibilidade de medicamentos e alternativas para a continuidade da vida sexual de idosos de ambos os sexos, porém a preocupação com a prevenção ainda apresenta-se insuficiente (Andrade, 2013).

Conclui-se que os resultados da AFE para os escores da EBAS com idosos não trouxeram apoio suficiente para a proposta teórica original. Recomenda-se que sejam realizadas novas pesquisas com 0 objetivo de adaptar ou criar uma medida de atitudes sexuais válida para essa população.

Os resultados desta pesquisa, assim como a revisão de literatura, revelaram que não há uma resposta simples para o problema complexo do comportamento de prevenção em idosos. Contudo, foi encontrado algum apoio para um modelo interpessoal, haja vista as pequenas evidências encontradas nas relações entre o risco às DSTs, o amor e as atitudes sexuais. Observou-se também que os motivos 
que levariam homens e mulheres idosos à exposição ao contágio de doenças por meio do sexo parecem diferentes. Entretanto, como a amostra utilizada nesta investigação é de conveniência, não se podem generalizar os resultados obtidos. Sugere-se que novas pesquisas com a população de idosos possam replicar e ampliar este estudo com variáveis disposicionais, tais como, autoestima e outras.

\section{Referências}

Abdo, C. H. N., Oliveira Jr, W. M., Scanavino, M. D. T., \& Martins, F. G. (2006). Disfunção erétil: resultados do estudo da vida sexual do brasileiro. Revista da Associação Médica Brasileira, 52(6), 424-429. http://dx.doi.org/10.1590/S010442302006000600023

Acker, M., \& Davis, M. H. (1992). Intimacy, Passion and Commitment in Adult Romantic Relationships: A Test of the Triangular Theory of Love. Journal of Social and Personal Relationships, 9, 21-50. doi: $10.1177 / 0265407592091002$

Adler, N., \& Hendrick, S. S. (1991). Relationships Between Contraceptive Behavior and Love Attitudes, Sex Attitudes, and Self-Esteem. Journal of Counseling \& Development, 70(2), 302308. doi: 10.1002/j.1556-6676.1991.tb01601.x

Ahmetoglu, G., Swami, V., \& Chamorro-Premuzic, T. (2010). The Relationship Between Dimensions of Love, Personality, and Relationship Length. Archives of Sexual Behavior, 39, 11811190. doi 10.1007/s10508-009-9515-5

Alferes, V. R. (1999. Escala de Atitudes Sexuais. In M. R. Simões, M. M. Gonçalves \& L. A. Almeida (Eds.), Testes e provas psicológicas em Portugal (vol. 2, pp. 131-148). Braga: SHO/APPORT.

Andrade, M. A. R. (2013). Velhos e felizes? Reflexões sobre a construção da velhice na contemporaneidade. Revista Política \& Trabalho, 1(38), 309-315.

Bodley-Tickell, A. T., Olowokure, B., Bhaduri, S., White, D. J., Ward, D., Ross, J. D. C., ... Goold, P. (2008). Trends in sexually transmitted infections (other than HIV) in older people: analysis of data from an enhanced surveillance system. Sex Transm Infect, 84, 312-317. doi: 10.1136/sti.2007.027847

Buss, D. M. (1998). Sexual strategies theory: Historical origins and current status. Journal of Sex Research, 35, 19-31. doi: $10.1080 / 00224499809551914$

Bussey, K., \& Bandura, A. (1999). Social cognitive theory of gender development and differentiation. Psychological Review, 106, 676-713. http://dx.doi.org/10.1037/0033-295X.106.4.676 
Carreño, M., \& Serrano, G. (1995). Analisis de instrumentos para la medida del amor. Revista de Psicologia Social, 10, 131-148.

Cassepp-Borges, V., \& Pasquali, L. (2012). Estudo nacional dos atributos psicométricos da Escala Triangular do Amor de Sternberg. Paidéia, 22, 21-31. doi: 10.1590/S0103863X2012000100004

Cassepp-Borges, V., \& Teodoro, M. (2007). Propriedades Psicométricas da versão brasileira da Escala Triangular do Amor de Sternberg. Psicologia: Reflexão e Crítica, 20, 513-522. doi: 10.1590/S010279722007000300020

Cohen, J. (1988). Statistical power analysis for the behavioral sciences. Hillsdale: Lawrence Erlbaum Associates.

Crawford, M., \& Popp, D. (2003). Sexual double standards: A review and methodological critique of two decades of research. Journal of Sex Research, 40, 13-26.

Dorneles Neto, J., Nakamura, A. S., Cortez, L. E. R., \& Yamaguchi, M. U. (2015). Doenças sexualmente transmissíveis em idosos: uma revisão sistemática. Ciência \& Saúde Coletiva, 20(12), 3853-3864.

http://dx.doi.org/10.1590/1413812320152012.17602014

Eagly, A. H., \& Wood, W. (1999). The origins of sex differences in human behavior: Evolved dispositions versus social roles. American Psychologist, 54, 408-423. http://dx.doi.org/10.1037/0003-066X.54.6.408

Felipe, L. A. P. V. (2012). Abertura à Experiência, Atitudes e Comportamentos Sexuais em Jovens do Ensino Superior. Dissertação de Mestrado. Faculdade de Medicina de Lisboa. Universidade de Lisboa, Portugal.

Finney, S. J., \& DiStefano, C. (2006). Non-normal and categorical data in structural equation modeling. In G. R. Hancock \& R. O. Mueller (Eds.), Structural equation modeling: A second course (pp. 269-314). Greenwich, CT: Information Age Publishing.

Garrett, D. (2014). Psychosocial barriers to sexual intimacy for older people. British Journal of Nursing, 23(6), 327-331.

Gradim, C. V. C., Sousa, A. M. M., \& Lobo, J. M. (2007). A prática sexual e o envelhecimento. Cogitare Enfermagem, 12(2), 20413. doi: http://dx.doi.org/10.5380/ce.v12i2

Graham, J. M. (2011). Measuring love in romantic relationships: A meta-analysis. Journal of Social and Personal Relationships, 28, 748-771. doi: $10.1177 / 0265407510389126$

Gouveia, V. V., Fonseca, P. N. da, Cavalcanti, J. P. N., Diniz, P. K. da C., \& Dória, L. C. (2009). Versão abreviada da Escala Triangular do Amor: Evidências de validade fatorial e consistência interna. Estudos de Psicologia, 14, 31-39. doi: 10.1590/S1413294X2009000100005 
Hall, A. C., Hendrick, S. S., \& Hendrick, C. (1991). Personal construct systems and love styles. International Journal of Personal Construct Psychology, 4(2), 137-155. doi: 10.1080/08936039108404769

Hatfield, E., \& Sprecher, S. (1986). Measuring passionate love in intimate relations. Journal of Adolescence, 9, 383-410.

Hendrick, C., \& Hendrick, S. S. (1986). A theory and method of love. Journal of Personality and Social Psychology, 50, 392-402. http://dx.doi.org/10.1037/0022-3514.50.2.392

Hendrick, C., \& Hendrick, S. S. (1989). Research on love: Does it measure up? Journal of Personality and Social Psychology, 56, 784-794. http://dx.doi.org/10.1037/0022-3514.56.5.784

Hendrick, C., Hendrick, S. S., \& Reich, D. A. (2006). The brief sexual attitudes scale. Journal of Sex Research, 43(1), 76-86. http://dx.doi.org/10.13072/midss. 230

Hendrick, S. S., \& Hendrick, C. (1987). Multidimensionality of Sexual Attitudes. The Journal of Sex Research, 23(4), 502-526. doi: $10.1080 / 00224498709551387$

Hendrick, S. S., \& Hendrick, C. (1995). Gender differences and similarities in sex and love. Personal Relationships, 2(1), 55-65. doi: 10.1111/j.1475-6811.1995.tb00077.x

Hendrick, S. S., \& Hendrick, C. (2002). Linking Romantic Love with Sex: Development of the Perceptions of Love and Sex Scale. Journal of Social and Personal Relationships, 19(3), 361-378. doi: $10.1177 / 0265407502193004$

Hendrick, S. S., Hendrick, C., Slapion-Foote, M. J., \& Foote, F. H. (1985). Gender differences in sexual attitudes. Journal of Personality and Social Psychology, 48(6), 1630-1642. http://dx.doi.org/10.1037/0022-3514.48.6.1630

Hernandez, J. A. E. (1999). Validação da estrutura da Escala Triangular do Amor: Análise fatorial confirmatória. Aletheia, 9, 15-25.

Hernandez, J. A. E. (2015). Evidências de Validade de Construto da Escala de Componentes do Amor. Psicologia: Teoria e Pesquisa, 31(2), 249-257. http://dx.doi.org/10.1590/010237722015021828249257

Hernandez, J. A. E. (2016). Análise fatorial exploratória e hierárquica da Escala Triangular do Amor. Avaliação Psicológica, 15(1), 1120. doi: 10.15689/ap.2016.1501.02

Horn, J. L. (1965). A Rationale and Test For the Number of Factors in Factor Analysis. Psychometrika, 30, 179-85. doi: 10.1007/BF02289447

Instituto Brasileiro de Geografia e Estatística (2014). Pesquisa Nacional de Amostra por Domicílios (PNAD) 2014. Texto completo

em: 
http://www.ibge.gov.br/home/estatistica/pesquisas/pesquisa_r esultados.php?id

Kreager, D. A., \& Staff, J. (2009). The sexual double standard and adolescent peer approval. Social Psychology Quarterly, 72, 143-164.

Laroque, M. F., Affeldt, A. B., Cardoso, D. H., Souza, G. L., Santana, M. G., \& Lange, C.(2011). Sexualidade do Idoso: comportamento para a prevenção de DST/AIDS. Revista Gaúcha de Enfermagem, 32(4), 774-780. http://dx.doi.org/10.1590/S1983-14472011000400019

Laros, J. A. (2005). O uso da análise fatorial: Algumas diretrizes para pesquisadores. In L. Pasquali (Org). Análise fatorial para pesquisadores (pp.163-184). Brasília: LabPAM.

Laurentino, N. R. S., Barboza, D., Chaves, G., Besutti, J., Bervian, S. A., \& Portella, M. R. (2006). Namoro na terceira idade e o processo de ser saudável na velhice: Recorte ilustrativo de um grupo de mulheres. Revista Brasileira de Ciências do Envelhecimento Humano, 3(1), 51-63.

Lorenzo-Seva, U. (2013). Why rotate my data using Promin? Technical Report. Department of Psychology, Universitat Rovira i Virgili, Tarragona.

Lorenzo-Seva, U., \& Ferrando, P. J. (2013). FACTOR 9.2: A Comprehensive Program for Fitting Exploratory and Semiconfirmatory Factor Analysis and IRT Models. Applied Psychological Measurement, 37, 497-498. doi: 10.1177/0146621613487794

Lloret-Segura, S., Ferreres-Traver, A., Hernández-Baeza, A., \& Tomás-Marco, I. (2014). El análisis factorial exploratorio de los ítems: una guía práctica, revisada y actualizada. Anales de Psicología, 30(3), 1151-1169. http://dx.doi.org/10.6018/analesps.30.3.199361

Milhausen, R. R., \& Herold, E. S. (2001). Reconceptualizing the sexual response: Physical and psychological factors that control our double standard. Journal of Psychology and Human Sexuality, 13, 63-83.

Neto, F. (2012). Perceptions of love and sex across the adult life span. Journal of Social and Personal Relationships, 29, 760775. doi: $10.1177 / 0265407512443638$.

Oliver, M. B., \& Hyde, J. S. (1993). Gender differences in sexuality: a meta-analysis. Psychological Bulletin, 114(1), 29-51. doi: 10.1037/0033-2909.114.1.29

Pascual, C. P. (2002). Sexualidade do idoso. São Paulo: Edições Loyola.

Petersen, J. L., \& Hyde, J. S. (2010). A meta-analytic review of research on gender differences in sexuality: 1993 to 2007. 
Psychological Bulletin, 136, 21-38. http://dx.doi.org/10.1037/a0017504

Petersen, J. L., \& Hyde, J. S. (2011). Gender Differences in Sexual Attitudes and Behaviors: A Review of Meta-Analytic Results and Large Datasets. Journal of Sex Research, 48(2-3), 149-165. doi: 10.1080/00224499.2011.551851

Rocha, E., \& Hernandez, J. A. E. (2002). Os jovens, os idosos e o Amor. In: XIV Salão de Iniciação Cientifica e XI Feira de Iniciação Científica, Universidade Federal do Rio Grande do Sul. Livro de Resumos. Porto Alegre: Gráfica da UFRGS, 802-802.

Rokach, A., \& Neto, F. (2005). Age, culture and the antecedents of loneliness. Social Behavior and Personality: An International Journal, 33, 477-494.

Rubin, Z. (1970). Measurement of romantic love. Journal of Personality and Social Psychology, 16, 265-273. doi: $10.1037 / \mathrm{h} 0029841$

Santos, A. F. D. M., \& Assis, M. D. (2011). Vulnerabilidade das idosas ao HIV AIDS despertar das políticas públicas e profissionais de saúde no contexto da atenção integral revisão de literatura. Revista Brasileira de Geriatria e Gerontologia, 14(1), 147-157.

Sternberg, R. J. (1986). A Triangular Theory of Love. Psychological Review, 93, 119-135. http://dx.doi.org/10.1037/0033295X.93.2.119

Sternberg, R. J. (1988). The triangle of love: Intimacy, passion, commitment. New York: Basic Books.

Sternberg, R. J. (1997). A construct-validation of a triangular love scale. European Journal of Social Psychology, 27, 313-335. doi: 10.1002/(SICI)1099-0992(199705)27:3<313: :AIDEJSP824>3.0.CO;2-4

Sternberg, R. J., \& Weis, K. (2006). The new psychology of love. Yale University Press.

Sumter, S. R., Valkenburg, P. M., \& Peter, J. (2013). Perceptions of love across the lifespan: Differences in passion, intimacy, and commitment. International Journal of Behavioral Development, 37(5), 417-427. doi: 10.1177/0165025413492486

Trudel, G., Boyer, R., Villeneuve, V., Anderson, A., Pilon, G., \& Bounader, J. (2008). The marital life and aging well program: effects of a group preventive intervention on the marital and sexual functioning of retired couples. Sexual and Relationship Therapy, 23(1), 5-23. doi: 10.1080/14681990701635061

Villar, F., Villamizar, D. J., \& López-Chivrall, S. (2005). Los componentes de la experiencia amorosa en la vejez: personas mayores y relaciones de pareja de larga duración. Revista Española de Geriatría y Gerontología, 40, 166-177. 


\section{Endereço para correspondência}

\section{Luiz Antônio da Silva}

Universidade Salgado de Oliveira - UNIVERSO

Rua Marechal Deodoro 217, $2^{\circ}$ Andar, Centro, CEP 24030-060, Niterói - RJ, Brasil

Endereço eletrônico: luizantoniopsicologo.ajuda@gmail.com

Lucia Helena de Freitas Pinho França

Universidade Salgado de Oliveira - UNIVERSO

Rua Marechal Deodoro 217, $2^{\circ}$ Andar, Centro, CEP 24030-060, Niterói - RJ, Brasil

Endereço eletrônico: lucia.franca@gmail.com

\section{José Augusto Evangelho Hernandez}

Universidade do Estado do Rio de Janeiro - UERJ

Rua São Francisco Xavier, 524, 10ªndar Sala 10028D, Maracanã, CEP 20550-900, Rio de Janeiro - RJ, Brasil

Endereço eletrônico: hernandez.uerj@gmail.com

Recebido em: 15/01/2016

Reformulado em: 18/10/2016

Aceito para publicação em: 21/10/2016

\section{Notas}

* Mestre em Psicologia pela Universidade Salgado de Oliveira.

** PhD em Psicologia pela University of Auckland/Nova Zelândia. Professora do PPG em Psicologia da Universidade Salgado de Oliveira.

*** Doutor em Psicologia do Desenvolvimento pela Universidade Federal do Rio Grande do Sul. Professor PPG em Psicologia Social da Universidade do Estado do Rio de Janeiro. 